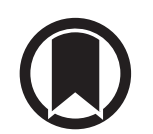

CrossMark

\section{Hearing loss with kanamycin treatment for multidrug-resistant tuberculosis in Bangladesh}

\author{
To the Editor:
}

World Health Organization (WHO) recommendations for multidrug-resistant tuberculosis (MDR-TB) include treatment for 8 months or more of an aminoglycoside, such as kanamycin, amikacin or capreomycin, or a shorter course of 4 months or more, depending on the susceptibility of the patient's Mycobacterium tuberculosis isolate to other drugs in the regimen [1]. Aminoglycosides can produce significant side-effects, including irreversible ototoxicity, estimated to occur in $25-60 \%$ of patients treated for MDR-TB, depending on methods of measurement [2-5]. Even if surviving to cure, MDR-TB treatment itself is impoverishing [6], while ototoxicity further limits a patient's ability to regain employment and may compound social isolation.

Clinical trials are underway with regimens that exclude aminoglycosides, but depend upon the activity of oral drugs including fluoroquinolones [7]. Yet in prior studies of MDR-TB in Bangladesh, we found high proportions of patients with $M$. tuberculosis isolates with resistance or borderline susceptibility to fluoroquinolones, while susceptibility to an aminoglycoside was retained [8]. Thus, we aimed to measure pure tone hearing thresholds at a wide range of frequencies prior to and during kanamycin treatment among MDR-TB patients in Bangladesh with a view to inform the development of an accurate effect size for interventional trials of kanamycin dose or duration reduction, or the future use of adjunct agents to prevent ototoxicity.

A prospective cohort of consecutive adults referred to the national MDR-TB hospital in Dhaka, Bangladesh, the National Institute of Diseases of the Chest and Hospital (NIDCH), were enrolled if initiating kanamycin for MDR-TB treatment from October 2015 to February 2016. The prospective participants were counselled regarding the follow-up schedule and the consenting participants were enrolled. All patients signed written informed consent and the protocol was approved by the NIDCH administration and the ethical review committees at the International Centre for Diarrhoeal Diseases Research, Bangladesh and the University of Virginia.

Patients initially received daily injectable kanamycin as part of a standardised WHO recommended second-line regimen for MDR-TB. Pure tone hearing threshold testing was performed by an audiologist in a dedicated room at baseline (i.e. prior to kanamycin treatment) in all patients, and at 2, 3, 6 and 8 months after treatment initiation. Threshold measurements for pure tones presented to each ear via air conduction were performed at 250,500,1000, 2000,4000, 6000 and $8000 \mathrm{~Hz}$, while those presented via bone conduction were at 500,1000, 2000 and $4000 \mathrm{~Hz}$. Baseline hearing loss was defined as threshold $>25 \mathrm{~dB}$ at any frequency. Acquired hearing loss (ototoxicity) was defined as: 1) pure tone threshold change (loss) $>20 \mathrm{~dB}$ compared to baseline at any frequency, 2) $>10 \mathrm{~dB}$ loss at two adjacent frequencies, or 3) any worsening of pure tone threshold at three consecutive frequencies in either ear [9]. Acquired hearing loss was further categorised as severe if $>70 \mathrm{~dB}$ loss at any frequency. Clinicians and patients were informed of pure tone test results for consideration of treatment alteration.

Pretreatment characteristics were compared among those with and without baseline hearing loss by Chi-square or Fisher's exact tests for categorical variables, and t-test for means of continuous variables. Kaplan-Meier survival analysis plotted acquired hearing loss as the event against the month of pure tone testing and right-censored at 8 months or the month of last testing.

@ERSpublications

High frequency hearing loss from kanamycin in MDR-TB treatment: not if, but when? http://ow.ly/dLQb30hPD2d

Cite this article as: Heysell SK, Ahmed S, Rahman MT, et al. Hearing loss with kanamycin treatment for multidrug-resistant tuberculosis in Bangladesh. Eur Respir J 2018; 51: 1701778 [https://doi.org/10.1183/ 13993003.01778-2017]. 
40 patients underwent baseline pure tone threshold testing; the mean \pm SD age was $29 \pm 11$ years, $22(55 \%)$ were male and six (13\%) had diabetes. Of 18 patients with prior TB treatment, 5 (28\%) received streptomycin for a prior re-treatment regimen (formerly known as category II). Baseline hearing loss was present in $18(45 \%)$ and while older mean age and prior TB treatment were more common among those with baseline hearing loss; these proportions were not statistically significant. The median kanamycin daily dose was $18.9 \mathrm{mg} \cdot \mathrm{kg}^{-1}$ (range $13.0-25.0 \mathrm{mg} \cdot \mathrm{kg}^{-1}$ ) and those with the lowest body mass index (BMI) received the highest daily doses $\left(\mathrm{R}^{2}=0.46, \mathrm{p}<0.001\right)$.

36 patients underwent follow-up pure tone testing and 28 (77.8\%) acquired new hearing loss, including 10 with baseline hearing loss. Acquired hearing loss was more common at higher frequencies, but in 11 patients (30.5\%) hearing loss occurred at or below $2000 \mathrm{~Hz}$. All patients with hearing loss below $2000 \mathrm{~Hz}$ also demonstrated acquired loss above $2000 \mathrm{~Hz}$. As early as 2 months after treatment, eight patients $(22 \%)$ acquired hearing loss. All patients with diabetes acquired hearing loss, and were significantly more likely to acquire loss early, with a mean event free survival of 2.8 months (95\% CI 1.2-4.4) compared to those without known diabetes (mean 4.7 months, 95\% CI 3.9-5.4; $\mathrm{p}=0.006$, Wilcoxon test equality of survival distribution) (figure 1).

Hearing loss during treatment for MDR-TB with kanamycin occurred in more than three quarters of all patients in this prospective cohort, one of the highest incidences reported to date. At the highest frequencies hearing loss was severe and progressed stepwise to involve frequencies considered critical for perception of conversational speech in nearly a third of patients.

While this increased frequency of monitoring at a wide range of thresholds may have detected cases missed by a less intensive approach [3-5], daily dosing strategies in this cohort were aggressive, in excess of WHO recommended daily dose of $15 \mathrm{mg} \cdot \mathrm{kg}^{-1}$ in 35 (87.5\%) of the initial 40 patients, and particularly so for those with the lowest BMI. Kanamycin is concentration-dependent in activity and the dose often correlates with the peak serum concentration, $\mathrm{C}_{\max }$, and the total serum exposure or area under the concentration curve (AUC) [10]. One MDR-TB treatment centre in the Netherlands that routinely uses serum therapeutic drug monitoring (TDM) to estimate $\mathrm{C}_{\max }$ and AUC has recently reported median doses as low as $6.5 \mathrm{mg} \cdot \mathrm{kg}^{-1}$ of kanamycin or amikacin that have prevented ototoxicity and preserved treatment efficacy [11].

Given the complexities of TDM, adjunct agents that may prevent toxicity also warrant study. Aminoglycosides form complexes with iron catalysing reactive oxygen species that result in basal hair cell destruction and basilar membrane apoptosis [12]. N-Acetylcysteine (NAC) has been used by some experts in co-administration with aminoglycosides for MDR-TB and in at least one meta-analysis has been found to prevent ototoxicity without additional harm [13]. Patients with diabetes progressed to hearing loss earlier than those without known diabetes and represent a subgroup which may be ideal for studying agents such as NAC. Prior age-matched retrospective studies in non-TB populations have found an association with diabetes and sensorineural hearing loss which correlated with worsening kidney function, leading to postulates of microangiopathic inner-ear processes [14]. Screening for diabetes at TB diagnosis

FIGURE 1 Kaplan-Meier curve of ototoxicity free survival among patients with known diabetes and those without.

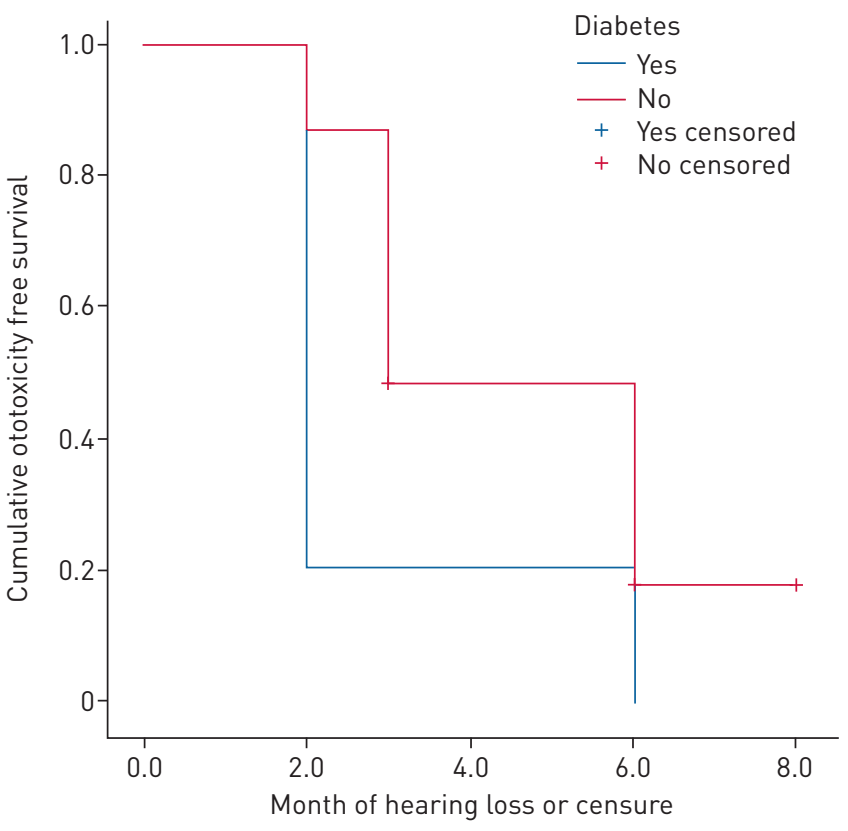


is now a broadly endorsed programmatic recommendation, but well-characterised studies of MDR-TB drug toxicities and pharmacodynamics across the diabetes disease spectrum are lacking [15]. Nevertheless, pending the results of new clinical trials, baseline audiometry and frequent follow-up monitoring appears warranted and can be dose tailored based on patient characteristics and preferably guided by TDM, with careful attention given to not exceeding the maximum WHO recommended dosage.

This study did not test for known, albeit rare, pharmacogenetic risk factors for aminoglycoside-induced ototoxicity [3]. In addition, the pattern of ototoxicity was not always definitively sensorineural, as would be expected for kanamycin, and differences in air and bone thresholds of more than $25 \mathrm{~dB}$ were suggestive of other middle ear pathology.

In conclusion, these findings provide a more accurate effect size of hearing loss in a high-risk cohort, and compel trials of reduced kanamycin exposure and/or adjunct agents to reduce ototoxicity.

Scott K. Heysell ${ }^{1,5}$, Shahriar Ahmed ${ }^{2,5}$, Md. Toufiq Rahman ${ }^{2}$, Md. Wahiduzzaman Akhanda ${ }^{4}$, A. Tucker Gleason ${ }^{3}$, Andrew Ebers ${ }^{1}$, Eric R. Houpt ${ }^{1}$ and Sayera Banu ${ }^{2}$

${ }^{1}$ Division of Infectious Diseases and International Health, University of Virginia, Charlottesvile, VA, USA. ${ }^{2}$ Infectious Disease Division, International Centre for Diarrhoeal Diseases Research, Bangladesh (icddr,b), Dhaka, Bangladesh. ${ }^{3}$ Dept of Otolaryngology, University of Virginia, Charlottesvile, VA, USA. ${ }^{4}$ National Tuberculosis Control Programme, Dhaka, Bangladesh. ${ }^{5}$ These authors contributed equally.

Correspondence: Sayera Banu, Infectious Diseases Division, icddr,b, 68, Shaheed Tajuddin Ahmed Sarani Mohakhali, Dhaka 1212, Bangladesh. E-mail: sbanu@icddrb.org

Received: May 202017 | Accepted after revision: Dec 212017

Support statement: This study was funded by the National Institutes of Health (NIH) via University of Virginia (R34 AI112371 and U01AI119954). Funding information for this article has been deposited with the Crossref Funder Registry.

Conflict of interest: None declared.

Acknowledgement: icddr,b acknowledges with gratitude the commitment of the National Institutes of Health to its research efforts. icddr,b is also grateful to the Governments of Bangladesh, Canada, Sweden and the UK for providing core/unrestricted support. The authors also express their sincere gratitude to the authority of NIDCH for their continued support to the studies conducted by icddr,b.

\section{References}

1 World Health Organization. WHO Treatment Guidelines for Drug-resistant Tuberculosis, 2016 Update. WHO/ HTM/TB/2016.04. Geneva, World Health Organization, 2016.

2 Sharma V, Bhagat S, Verma B, et al. Audiological evaluation of patients taking kanamycin for multidrug resistant tuberculosis. Iran J Otorhinolaryng 2016; 28: 203-208.

3 Harris T, Bardien S, Schaaf S, et al. Aminoglycoside-induced hearing loss in HIV-positive and HIV-negative multidrug- resistant tuberculosis patients. South Afr Med J 2012; 102: 363-366.

4 Modongo C, Sobota R, Kesenogile B, et al. Successful MDR-TB treatment regimens including amikacin are associated with high rates of hearing loss. BMC Infect Dis 2014; 14: 1-9.

5 Törün T, Güngör G, Özmen I, et al. Side effects associated with the treatment of multidrug-resistant tuberculosis. Int J Tuberc Lung Dis 2005; 9: 1373-1377.

6 Ayé $\mathrm{R}$, Wyss $\mathrm{K}$, Abdualimova $\mathrm{H}$, et al. Household costs of illness during different phases of tuberculosis treatment in Central Asia: a patient survey in Tajikistan. BMC Public Health 2010; 10: 18.

7 Moodley R, Godec TR. STREAM Trial Team. Short-course treatment for multidrug-resistant tuberculosis: the STREAM trials. Eur Respir Rev 2016; 25: 29-35.

8 Heysell SK, Ahmed S, Mazidur SMM, et al. Quantitative second-line drug-susceptibility in patients treated for multidrug-resistant tuberculosis in Bangladesh: implications for regimen choice. PLoS One 2015; 10: e0116795.

9 American Speech-Language-Hearing Association audiologic management of individuals receiving cochleotoxic drug therapy. Guidelines for audiologic management of individuals receiving cochleotoxic drug therapy. ASHA 1994; 34: 11-19.

10 Modongo C, Pasipanodya JG, Magazi BT, et al. Artificial intelligence and amikacin exposures predictive of outcomes in multidrug-resistant tuberculosis patients. Antimicrob Agents Chemother 2016; 60: 5928-5932.

11 van Altena R, Dijkstra JA, van der Meer ME, et al. Reduced chance of hearing loss associated with therapeutic drug monitoring of aminoglycosides in the treatment of multidrug-resistant tuberculosis. Antimicrob Agents Chemother 2017; 60: e01400-16.

12 Duggal P, Sarkar M. Audiological monitoring of multi-drug resistant tuberculosis patients on aminoglycoside treatment with long term follow-up. BMC Ear Nose Throat Disorders 2007; 7: 2.

13 Kranzer K, Elamin WF, Cox H, et al. A systematic review and meta-analysis of the efficacy and safety of $\mathrm{N}$-acetylcysteine in preventing aminoglycoside-induced ototoxicity: implications for the treatment of multidrug-resistant TB. Thorax 2015; 70: 1070-1107.

14 Kakarlapudi V, Sawyer R, Staecker H. The effect of diabetes on sensorineural hearing loss. Otolog Laryngol 2003; 24: $382-386$.

15 Munoz-Torrico M, Caminero-Luna J, Migliori GB, et al. Diabetes is associated with severe adverse events in multidrug-resistant tuberculosis. Arch Bronconeumol 2017; 53: 245-250. 